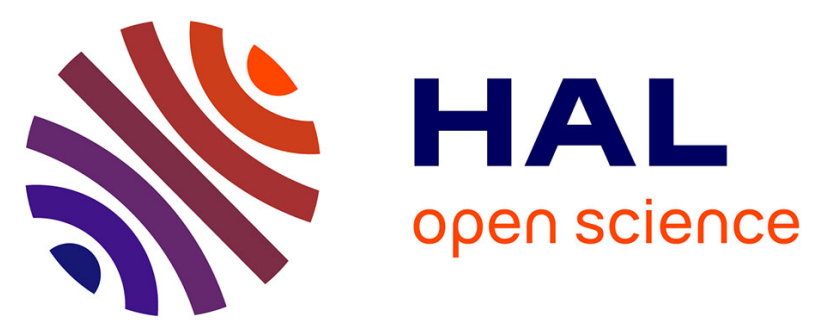

\title{
Gestion de l'environnement et valorisation des produits de l'élevage de montagne au regard de la théorie des communs
}

\author{
Philippe Fleury, Marie M. Houdart, Jacques Lasseur, Virginie Baritaux, \\ Carole Chazoule, Christian Corniaux, Martine Napoleone, René \\ Poccard-Chapuis
}

\section{To cite this version:}

Philippe Fleury, Marie M. Houdart, Jacques Lasseur, Virginie Baritaux, Carole Chazoule, et al.. Gestion de l'environnement et valorisation des produits de l'élevage de montagne au regard de la théorie des communs. Géocarrefour - Revue de géographie de Lyon, 2018, 92 (3), 19 p. 10.4000/geocarrefour.10925 . hal-02121206

\section{HAL Id: hal-02121206 \\ https://hal.uca.fr/hal-02121206}

Submitted on 25 May 2020

HAL is a multi-disciplinary open access archive for the deposit and dissemination of scientific research documents, whether they are published or not. The documents may come from teaching and research institutions in France or abroad, or from public or private research centers.
L'archive ouverte pluridisciplinaire HAL, est destinée au dépôt et à la diffusion de documents scientifiques de niveau recherche, publiés ou non, émanant des établissements d'enseignement et de recherche français ou étrangers, des laboratoires publics ou privés. 


\section{Géocarrefour}

$92 / 3 \mid 2018$

L'élevage dans tous ses territoires (dans les pays développés)

\section{Gestion de l'environnement et valorisation des produits de l'élevage de montagne au regard de la théorie des communs}

Environmental management and enhancing the value of mountain livestock products using the "commons" theory

Philippe Fleury, Marie Houdart, Jacques Lasseur, Virginie Baritaux, Carole Chazoule, Martine Napoléone et René Poccard-Chapuis

\section{OpenEdition}

\section{Journals}

Édition électronique

URL : http://journals.openedition.org/geocarrefour/10925

DOI : $10.4000 /$ geocarrefour.10925

ISSN : 1960-601X

\section{Éditeur}

Association des amis de la Revue de géographie de Lyon

Ce document vous est offert par INRA Institut National de la Recherche Agronomique

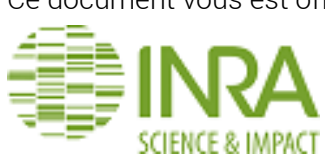

Référence électronique

Philippe Fleury, Marie Houdart, Jacques Lasseur, Virginie Baritaux, Carole Chazoule, Martine

Napoléone et René Poccard-Chapuis, "Gestion de l'environnement et valorisation des produits de

l'élevage de montagne au regard de la théorie des communs », Géocarrefour [En ligne], 92/3 | 2018, mis en ligne le 15 décembre 2018, consulté le 11 février 2019. URL : http://journals.openedition.org/ geocarrefour/10925; DOI : 10.4000/geocarrefour.10925

Ce document a été généré automatiquement le 11 février 2019.

(C) Géocarrefour 


\title{
Gestion de l'environnement et valorisation des produits de l'élevage de montagne au regard de la théorie des communs
}

\author{
Environmental management and enhancing the value of mountain livestock \\ products using the "commons" theory \\ Philippe Fleury, Marie Houdart, Jacques Lasseur, Virginie Baritaux, \\ Carole Chazoule, Martine Napoléone et René Poccard-Chapuis
}

Cet article s'appuie sur des travaux de recherche conduits dans le projet MOUVE (les interactions Elevage et Territoire dans la mise en mouvement de l'intensification écologique) financé par l'agence nationale de la recherche (ANR-2010-STRA-005-01).

\section{Introduction}

\section{Envisager globalement gestion des ressources naturelles et valorisation des produits de l'élevage}

1 Les débats sur l'avenir de l'élevage intègrent aujourd'hui tout à la fois des préoccupations relatives à l'alimentation humaine (FAO, 2009), au revenu et au maintien des populations d'éleveurs (Ryschawy et al., 2015) comme d'autres concernant la préservation de l'environnement, la limitation du changement climatique ou encore les divers rôles sociaux, culturels et patrimoniaux de l'élevage (Dumont et al., 2016). Ces considérations sont de plus en plus fréquemment posées dans une perspective territoriale. L'élevage, notamment celui d'herbivores, n'est en effet pas réductible à une activité sectorielle et au monde professionnel des éleveurs. Il est au contraire fortement lié à des filières de transformation et de mise en marché des produits, mais est aussi ancré dans une société et un espace local où il fournit des produits et services multiples (Rubino et al., 2006). Ces 
services sont à la fois écologiques, sociaux et économiques (Dumont et al., 2016), l'élevage contribuant à l'identité et au développement des territoires. L'importance de l'élevage dans le développement des zones rurales est particulièrement mise en exergue dans les régions aux conditions naturelles difficiles comme les montagnes et les zones sèches (FAO, 2009; Otte et al., 2012). Les formes d'élevage qui y sont présentes relèvent souvent bien moins du modèle agro-industriel que du pastoralisme c'est-à-dire d'un élevage extensif avec un recours important aux pâturages et aux parcours.

Dans ces débats, les nouveaux rôles et fonctions de l'élevage sont fréquemment abordées en séparant ce qui relève de la gestion de l'espace et des écosystèmes de ce qui concerne la mise en marché et la valorisation des produits. On retrouve ainsi une séparation entre agriculture et alimentation fréquente, dans les disciplines scientifiques comme dans les politiques publiques (Lamine et Chiffoleau, 2012). Ainsi, si de nombreuses méthodes d'analyse de la durabilité permettent l'évaluation des performances de l'agriculture, rares sont ceux qui prennent en compte globalement les activités de production, mais aussi de transformation, de distribution et de commercialisation des produits de l'agriculture (Marshall, 2015).

3 Dans cet article, nous nous proposons, dans une perspective de géographie économique et sociale, de conduire une analyse qualitative des mutations des liens entre élevage et territoire. Pour cela, nous envisageons de façon globale les transformations de l'élevage en saisissant, sur un même territoire, les interactions entre les dynamiques visant à une meilleure valorisation des produits et celle concernant une amélioration des contributions de l'élevage au fonctionnement écologique des agro-écosystèmes. Il s'agit donc de traiter des questions soulevées par l'articulation de dimensions portées usuellement par des organisations conçues pour la mise en marché des produits et d'actions impliquant des gestionnaires du territoire et de l'environnement.

\section{L'élevage comme bien commun?}

4 Ces questions sont très vastes et nous avons privilégié deux angles de lecture. Tout d'abord nous avons un regard particulier sur le mouvement actuel de reterritorialisation de certaines productions agricoles dans une perspective de différenciation et de valorisation économique. Ensuite, notre regard se porte sur l'action collective, c'est-àdire sur les normes et les règles que les acteurs élaborent pour coopérer. Nous nous intéressons aux modalités et aux conditions dans lesquelles des dynamiques collectives se construisent. Dans cet article, nous nous appuyons sur les concepts associés de bien commun et d'action collective (Ostrom, 1990) dans leur capacité à renouveler les approches des processus de changement de l'élevage. «L'école des commons » (Ostrom, 1990 et 2008) a en effet montré que dans certains cas la gestion de certaines ressources naturelles pouvait faire l'objet d'une auto-organisation collective durable et être ainsi gérées en « bien commun » ou en « ressource commune ».

5 Ces travaux ont porté initialement sur des biens communs simples, comme la gestion par des sociétés agricoles ou pastorales de ressources naturelles locales. Cette approche s'élargit aujourd'hui à des biens communs complexes (" complex commons») comme la gestion multi-usage d'une ressource naturelle ou celle nécessairement globale du changement climatique. Le terme de commun complexe renvoie soit à une gestion pour plusieurs usages soit à une gestion simultanée de plusieurs biens communs (Wilson, 2015). Cette gestion de communs complexes est souvent assurée par des groupes d'acteurs 
différents, mais plus ou moins interconnectés. A l'origine, la théorie d'Ostrom, bien que distinguant la nature de la ressource de son mode de gouvernance, établissait cependant une relation forte entre nature de la ressource et mode de gouvernance. Aujourd'hui le statut de bien commun d'une ressource est vu avant tout comme une construction sociale. La notion de bien commun est largement mobilisée au-delà de la seule gestion de ressources naturelles. Ainsi, les connaissances et leur diffusion numérique font l'objet dans certaines conditions d'une gestion en bien commun (Knowledge commons ou communs de la connaissance) (Hess and Ostrom, 2006).

Dans cet article, nous mobilisons le cadre des communs en privilégiant une approche constructiviste des biens communs et de l'action collective et en considérant l'élevage et le territoire comme des entités en interaction. Pour ce faire, nous déplaçons la perspective classique d'analyse de la gestion de l'espace pastoral comme une ressource commune en posant plus globalement la question des transformations de l'élevage sous l'angle d'une éventuelle construction d'une gestion de celui-ci en «bien commun complexe». En nous appuyant sur le cadre analytique des communs nous chercherons à voir s'il est possible d'interpréter certaines formes de gouvernance de l'élevage comme des formes de gestion en bien commun complexe, renvoyant à des usages et des rôles multiples (productifs, environnementaux, sociaux) et à des formes de collaboration multiacteurs et multi-niveaux. Pour aborder cette question nous nous situons au niveau territorial, vu comme l'échelle de la mise en valeur des espaces pastoraux et de la construction de projets de valorisation des produits.

7 Notre contribution s'appuiera sur une analyse comparée de quatre études de cas françaises situées dans des régions aux conditions physiques et climatiques qualifiées de difficiles: montagne et arrière-pays méditerranéen. L'élevage de ces territoires est engagé, selon des degrés et des modalités divers, dans des actions de développement local s'appuyant sur une stratégie de valorisation de ses produits comme dans d'autres actions en faveur de la préservation de l'environnement.

Dans le plan de cet article nous veillons à associer présentation concrète des enjeux et des dynamiques en cours dans les études de cas et discussion générique. Dans un premier temps nous présentons le cadre théorique des biens communs. Nous rendons compte ensuite des principaux résultats de chaque étude de cas avant d'en faire une analyse comparée permettant de discuter les intérêts et les limites théoriques et opérationnels d'une approche de l'élevage en bien commun.

\section{Cadre théorique}

\section{LES PÂTURAgES COLLECTIFS À L'ORIgINE dE LA THÉORIE dES COMMUNS}

Ce sont les travaux de Hardin (1968) qui ont ouvert le champ de recherche sur les communs. Dans cet article de référence, il s'est interrogé sur les limites d'un usage collectif par rapport à la privatisation ou à une réglementation étatique forte en s'appuyant sur l'exemple d'un pâturage collectif. Depuis, la théorie des communs et ses mises à l'épreuve dans de multiples études de cas (Ostrom 1990 ; Poteete et al., 2010) ont démontré que, dans certains cas, des acteurs sont capables de négocier et de respecter des accords leur permettant une gestion collective durable d'un bien commun. Par opposition aux conclusions théoriques d'Hardin, l'action collective apparaît comme une alternative 
crédible à la privatisation ou à la réglementation étatique. L'action collective, c'est-à-dire la négociation de normes et de règles cadrant les actions individuelles et collectives permet de mettre en place un mode de gestion évitant la surutilisation (over-harvesting) $\mathrm{du}$ bien commun. Les biens communs sont des ressources ne faisant pas l'objet d'une appropriation privée mais dont l'usage peut en compromettre la durabilité. La théorie économique définit les biens communs comme des biens dotés d'attributs de non exclusivité (on ne peut exclure personne de son usage) et de rivalité (l'usage des uns peut limiter ceux des autres). La théorie des communs a été utilisé avec succès, dans l'analyse et l'aide à l'action, pour une grande diversité de ressources naturelles: zones de pêche, eau et irrigation, plantation et gestion multifonctionnelle des forêts, espaces pastoraux (Poteete et al., 2010).

\section{Critiques et développements récents de la théorie des communs}

10 La théorie initiale de la gouvernance des communs a fait l'objet de deux critiques principales (Mosse, 2008 ; Baron et al., 2011 ; Dardot et Laval, 2014). La première concerne la distinction faite par Ostrom (1990) entre la nature du bien et son régime de gestion. Les ressources communes étudiées (les common pool ressources) sont avant tout des ressources naturelles. Elles sont apparentées à un réservoir dans lequel on puise et dont il suffirait de maintenir le niveau par de bonnes pratiques de gestion collectives (la gestion en bien commun). Mais, d'autres ressources communes, n'ont rien de naturelles, ce sont leurs règles de gouvernance qui déterminent ce statut. Il en est ainsi des connaissances dont la diffusion numérique peut-être gérée en bien commun (Hess and Ostrom, 2006) mais aussi des pâturages collectifs de Hardin qui ne sont pas un bien naturel figé dans lequel une société pastorale puiserait plus ou moins raisonnablement. Les pâturages collectifs sont bien construits, comme ressource écologique et comme réalité sociale par des règles d'usages ayant une historicité. Il est aujourd'hui largement admis que la gestion en bien commun est une construction sociale assez indépendante de la nature du bien (Wilson, 2015). Ainsi, Fournier et al. (2018) ont montré que selon leurs modalités de gestion les indications géographiques (IG) pouvaient être l'objet d'une gouvernance en bien commun ou en bien de club. La gestion en bien commun de certaines IG correspond à une forte coordination entre acteurs assurant leur durabilité. Dans la gestion en bien de club, l'usage de l'IG par l'un ne limite pas son usage pour les autres membres du club. Ceci se traduit par de faibles investissements dans des actions de préservation et de valorisation de l'IG, celle-ci n'étant pas vue comme à préserver, ce qui a terme peut menacer sa pérennité. Pour les IG, mais aussi pour le paysage, ou la vie villageoise (Van der Ploeg et al., 2012) c'est bien le partage collectif de règles d'usage qui fonde l'existence de la ressource commune.

11 La seconde critique de la théorie des communs concerne le comportement des acteurs, vu de façon très simplifiée (Baron et al., 2011). Elle se focalise en effet sur le comportement individuel d'acteurs cherchant à maximiser leur bénéfice personnel. L'approche se situe dans le champ de la rationalité individuelle : c'est l'anticipation des bénéfices attendus qui conduit les acteurs d'E. Ostrom à s'impliquer dans la mise en place et le respect d'institutions vouées à la préservation de la ressource commune. Ainsi, même si dans le cadre théorique d'Ostrom la confiance et les apprentissages sont déterminants (Berge, 2011) il peine à prendre en compte les rapports de pouvoir et les conflits (Clément, 2013). Maximisation de l'intérêt individuel et collectif sont vus comme liés. La communauté 
gestionnaire du bien commun est supposée homogène, sans hiérarchie et la théorie bute dans sa capacité à saisir les phénomènes d'exclusion ou d'accès inégalitaire à la ressource. Elle a au final tendance à produire une représentation dépolitisée de la situation (Stojanovic et al., 2016).

Les développements théoriques actuels (De Moor, 2011 ; Berge 2011) autour des notions d'action collective et de bien commun abordent des ressources complexes (complex commons). Ils s'intéressent à des comportements individuels plus élaborés que la seule rationalité économique en intégrant les apprentissages liés à une dynamique sociale. Le statut de bien commun ne concerne plus seulement des réalités naturelles externes aux sociétés (Dietz et al., 2003). Il correspond non seulement à des ressources naturelles mais aussi à des ressources cognitives, des savoirs techniques et relationnels, des valeurs. L'analyse des interactions sociales et des normes nécessaires à la construction, à l'appropriation et à la gestion d'une ressource commune s'affine également.

13 Cette conception renouvelée et ouverte à de nouveaux développements de la notion de ressource commune permet d'envisager de ne plus traiter de façon isolée la gestion de l'espace pastoral comme ressource collective d'une part et, d'autre part la construction de ressources partagées dans les activités de valorisation des produits de l'élevage. En associant ces deux angles d'analyse nous cherchons à traiter des questions suivantes: quelles sont les modalités de gouvernance de l'élevage dans les territoires? Peut-on considérer que certaines d'entre-elles relèvent d'une négociation et d'une gestion en "bien commun»? Quelles sont les composantes de l'élevage prises en compte et y a-t-il gouvernance d'un "complex common" envisageant globalement espace pastoral et produits de l'élevage? Sur quels types d'arrangements institutionnels entre acteurs, publics et privés, cette gouvernance repose-t-elle?

\section{Méthodologie de terrain et d'analyse}

14 Nous nous appuyons sur l'étude d'initiatives de développement local de l'élevage visant la valorisation et la différenciation collective des produits dans un contexte où les questions environnementales sont fortement présentes. Les études de cas concernent des territoires où les systèmes d'élevage pastoraux dominent et où l'objectif de valorisation des productions se traduit souvent par une augmentation de leur volume et par une intensification des usages de l'espace par l'élevage. Ces études de cas concernent également des territoires où les enjeux de développement durable et de préservation de l'environnement sont forts. Nous avons ainsi travaillé dans deux Parcs Naturels régionaux (PNR), un Parc national et un territoire de Pays. De ce fait des tensions peuvent se faire jour entre dimensions productives et gestion des problèmes environnementaux liés à l'élevage (Baritaux et al., 2016).

15 Les cas sélectionnés sont divers des points de vue du contexte socio-économique et institutionnel, de leur gouvernance et des acteurs impliqués. Ils rendent compte de la diversité des initiatives collectives de reterritorialisation des systèmes agro-alimentaires identifiée par Ilbery et al. (2005). Ces auteurs montrent qu'il existe deux grands types de rationalités dans les liens établis entre le lieu, le produit et ses conditions de production. La première qualifiée de rationalité territoriale privilégie les liens entre le produit et son lieu de production, le terroir. La seconde est une rationalité critique dans laquelle l'attention est donnée aux dimensions environnementales, sociales ou économiques des modes de production et de transformation d'un produit. Nos deux premières études 
relèvent surtout d'une rationalité territoriale et les deux suivantes d'une rationalité critique. Cependant nous verrons, au fil du texte, que les frontières entre ces rationalités ne sont pas étanches. Ces quatre études de cas sont les suivantes:

1. AOC Bleu du Vercors Sassenage dans le parc naturel régional (PNR) du Vercors ;

2. Le fromage AOP Pélardon, dans les Cévennes

3. Dynamique de l'élevage ovin en pays Asses-Verdon-Vaïre-Var ;

4. Filière Engagement Qualité Carrefour Bleu et Fourme d'Ambert, PNR du Livradois-Forez .

Ces démarches ont été qualifiées des points de vue de leur trajectoire et de leurs objectifs, des acteurs impliqués et des formes de gouvernance. Ces quatre études de cas sont présentées de façon synthétique dans le tableau 1 avant d'être exposées en détail dans le texte.

Les informations recueillies sur les différentes études de cas s'appuient sur une analyse des documents les concernant (diagnostics et études locaux divers, présentations des projets) et, pour l'essentiel sur des entretiens semi-directifs (Baritaux et al., 2016). Sur chaque territoire, une équipe de chercheurs a interviewé 20 à 25 acteurs représentatifs des personnes et des organisations concernées par l'initiative : agriculteurs, entreprises privées et coopératives assurant la transformation et la distribution des produits, gestionnaires de l'environnement et associations de protection de la nature, conseillers agricoles, élus et agents des collectivités locales, administrations.

Les entretiens ont abordé les thématiques suivantes: visions du contexte local, des enjeux, des objectifs et de la gouvernance de l'initiative, histoire de celle-ci (période d'émergence, phases clés, difficultés...) et liens éventuels avec d'autres projets du territoire, acteurs impliqués (qui, combien, rôles dans l'initiative, ressources matérielles ou immatérielles (écologique, cognitive, technologique...) sur lesquelles l'initiative s'appuie et effets positifs et négatifs envisagés. Une attention particulière a été attachée aux questions environnementales et à leurs éventuels liens avec la valorisation des produits de l'élevage: évolutions récentes des pratiques d'élevage et conséquences environnementales, controverses environnementales liées à l'élevage, politiques publiques environnementales, stratégies de valorisation des produits, utilisation d'attributs environnementaux dans celles-ci.

Tableau 1 : Description des études de cas

\begin{tabular}{|c|c|c|c|}
\hline Initiative & Contexte et enjeux & $\begin{array}{l}\text { Les ressources } \\
\text { communes }\end{array}$ & $\begin{array}{l}\text { Acteurs et formes de } \\
\text { gouvernance }\end{array}$ \\
\hline $\begin{array}{ll}\text { Bleu } & \mathrm{du} \\
\text { Vercors } & \\
\text { Sassenage } & \end{array}$ & 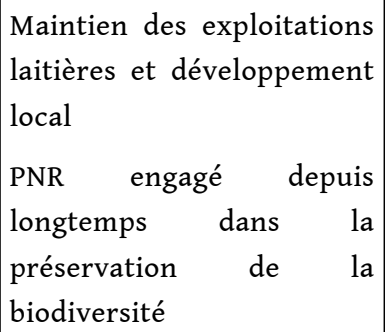 & $\begin{array}{l}\text { Bleu du Vercors } \\
\text { Sassenage } \\
\text { Prairies et } \\
\text { parcours (paysage } \\
\text { et biodiversité) }\end{array}$ & $\begin{array}{l}\text { Plusieurs lieux de } \\
\text { gouvernance (valorisation } \\
\text { des produits, } \\
\text { 'environnement, conseil } \\
\text { technique aux éleveurs), le } \\
\text { PNR essaie de faire du lien } \\
\text { entre eux }\end{array}$ \\
\hline
\end{tabular}




\begin{tabular}{|c|c|c|c|}
\hline $\begin{array}{lr}\text { Le } & \text { Pélardon, } \\
\text { fromage } & \text { AOP } \\
\text { dans } & \text { les } \\
\text { Cévennes } & \end{array}$ & $\begin{array}{l}\text { Valorisation du fromage de } \\
\text { chèvre Pélardon et } \\
\text { maintien d'une activité } \\
\text { d'élevage dans un milieu } \\
\text { difficile marqué par la } \\
\text { déprise agricole. } \\
\text { Depuis quelques années } \\
\text { enjeu partagé de lier } \\
\text { élevage, développement } \\
\text { local et paysage }\end{array}$ & $\begin{array}{l}\text { Le Pélardon avec } \\
\text { son antériorité } \\
\text { historique. } \\
\text { Le pâturage } \\
\text { Une entité } \\
\text { territoriale et ses } \\
\text { paysages }\end{array}$ & $\begin{array}{l}\text { AOP Pélardon, coopérative } \\
\text { des Cévennes, fermiers, } \\
\text { affineurs } \\
\text { Appui des organisations de } \\
\text { développement agricole, du } \\
\text { Parc national des Cévennes et } \\
\text { des élus locaux }\end{array}$ \\
\hline $\begin{array}{l}\text { Elevage ovin } \\
\text { en Pays Asses- } \\
\text { Verdon-Vaïre- } \\
\text { Var }\end{array}$ & $\begin{array}{l}\text { Importance de l'élevage } \\
\text { dans l'activité économique } \\
\text { du pays et dans la } \\
\text { structuration du paysage. } \\
\text { Le Pays a le statut } \\
\text { d'association 1901, il } \\
\text { regroupe communes et } \\
\text { intercommunalités autour } \\
\text { d'un projet de territoire } \\
\text { partagé. Il développe en } \\
\text { particulier des actions pour } \\
\text { conforter l'élevage et } \\
\text { valoriser ses produits } \\
\text { auprès des touristes: } \\
\text { maison de pays, label } \\
\text { "pays gourmand ", } \\
\text { ouverture du milieu boisé à } \\
\text { des fins pastorales }\end{array}$ & $\begin{array}{l}\text { Terroir avec des } \\
\text { produits agricoles } \\
\text { traditionnels } \\
\text { Paysage local } \\
\text { Les ressources } \\
\text { fourragères }\end{array}$ & $\begin{array}{l}\text { Comité de développement du } \\
\text { pays et un chargé de mission, } \\
\text { éleveurs, restaurateurs, } \\
\text { propriétaires fonciers } \\
\text { Gouvernance locale, charte } \\
\text { d'agriculture durable, } \\
\text { contractualisations }\end{array}$ \\
\hline $\begin{array}{|ll|}\text { Filière } & \\
\text { Engagement } & \\
\text { Qualité } & \\
\text { Carrefour } & \\
\text { Bleu et } & \\
\text { Fourme } & \\
\text { d'Ambert, } \\
\text { PNR du } \\
\text { Livradois- } \\
\text { Forez }\end{array}$ & $\begin{array}{l}\text { Assurer le maintien et le } \\
\text { revenu des éleveurs bovins } \\
\text { laitiers extensifs Entretien } \\
\text { des paysages sujets au } \\
\text { boisement }\end{array}$ & $\begin{array}{l}\text { Bleu et Fourme } \\
\text { d'Ambert } \\
\text { Prairies } \\
\text { Systèmes de } \\
\text { production } \\
\text { agricoles «tout } \\
\text { foin» }\end{array}$ & $\begin{array}{l}\text { Laiterie, enseigne de la } \\
\text { grande distribution, une } \\
\text { vingtaine d'éleveurs. } \\
\text { Contractualisation autour } \\
\text { d'un cahier des charges plus } \\
\text { contraignant que l'AOP sur } \\
\text { les pratiques de production } \\
\text { (ensilage interdit) et de } \\
\text { transformation }\end{array}$ \\
\hline
\end{tabular}

\section{Les études de cas}

\section{AOC Bleu du Vercors Sassenage dans le parc naturel régional du Vercors (France)}

Dans le parc naturel régional du Vercors, au début des années 1990, le modèle d'intensification de l'agriculture issu de la plaine montre ses limites et de nombreuses 
exploitations ont disparu. L'idée de protéger le «bleu du Vercors Sassenage » par une AOC voit le jour. Il s'agit de conforter les exploitations agricoles et les emplois dans une perspective de développement local. Pour cela, le cahier des charges doit permettre de différencier le produit, de le spécifier, mais il doit aussi réunir le plus grand nombre et permettre à tous d'adhérer. En 1998, l'AOC «Bleu du Vercors Sassenage » est reconnue. Les spécifications de l'AOC reposent sur trois dimensions: seules trois races traditionnelles de la région sont autorisées, les vaches doivent être nourries essentiellement à base d'herbe et de foin venant de l'aire de l'AOC et la fabrication du fromage s'appuie sur des procédés spécifiques justifiés par son histoire.

$\mathrm{Au}$ milieu des années 2000, force est de constater que les résultats économiques ne sont pas au rendez-vous. La production de Bleu ne se développe pas, le fromage reste peu connu. La coopérative qui transforme le bleu peine à vivre, les producteurs laitiers ne perçoivent pas de plus-value du fait de l'AOC. Seuls les producteurs fermiers arrivent, via la vente directe, à valoriser correctement leur lait. Pour faire face à ces difficultés, la décision est prise de développer une gamme de fromages et de produits agricoles autour du Bleu dont des produits biologiques. Différentes formes de vente directe (à la ferme, dans les restaurants et boutiques locales, dans des magasins dans les villes proches) et de promotion des produits, dont la fête du Bleu du Vercors, sont mises en place. Cette dynamique porte ses fruits et depuis plusieurs années la rémunération du prix du lait pour les producteurs se situe au-dessus de la moyenne nationale.

21 La thématique de l'environnement est présente dans le Vercors depuis le début des années 90. En 1991, le Vercors est un des territoires pionniers qui s'engage dans la signature de contrats agri-environnementaux avec des éleveurs volontaires. L'objectif est de préserver la biodiversité par exemple en retardant la fauche. Ces mesures sont diversement accueillies par les éleveurs mais conçues sans eux et visant des espaces ayant peu d'importance productive, elles ne modifient pas leurs pratiques. Aujourd'hui, la question de l'environnement est intégrée par tous les acteurs mais selon des visions et des modalités différentes.

Ainsi, la gestion des prairies divise producteurs fermiers, producteurs laitiers livrant leur lait à la coopérative et environnementalistes. Pour les fermiers, une gestion extensive des prairies permanentes, même si elle se traduit par une réduction de la production est essentielle. Elle permet la présence de nombreuses fleurs et c'est avec la race bovine locale, la Villarde et la fabrication au lait cru un des symboles forts de leur identité et de la spécificité du Bleu. Les éleveurs laitiers, au lait moins rémunéré, sont soucieux de réduire les coûts d'alimentation des troupeaux en intensifiant la production des prairies : il s'agit, sans recours à la "chimie ", de s'appuyer sur certaines pratiques adaptées à un contexte montagnard, comme la valorisation des fumiers et lisiers et le recours aux prairies temporaires. La biodiversité passe au second rang par rapport à la productivité des prairies.

Pour les environnementalistes, la référence est la biodiversité des prairies permanentes, à la fois élément à protéger et facteur de production agricole car les prairies biodiverses sont considérées comme procurant un fourrage de meilleure qualité. C'est un discours qu'entendent les fermiers mais qui se heurte à la logique et aux contraintes des éleveurs laitiers plus nombreux. Cette controverse sur ce que doivent être les pratiques d'élevage est forte. Elle se fait dans un contexte où les échanges entre parties prenantes restent limités, préservation de l'environnement et valorisation des produits se discutant dans des instances différentes. Certains acteurs, en particulier le chargé de mission agriculture 
du PNR, sont présents dans ces différents lieux et essaient, non sans difficultés, de faire le lien et d'assurer une cohérence des visions et des actions.

\section{Le Pélardon, fromage AOP dans les Cévennes}

Dans les Cévennes et les garrigues du Languedoc, les éleveurs caprins engagent en 1990 une procédure de demande de reconnaissance en $A O C$ de leur fromage de chèvre, le Pélardon. Celle-ci aboutit en 2000 à une AOC reconnue en AOP en 2001. La construction de l'AOP a fédéré un ensemble d'acteurs, producteurs fermiers et laitiers, transformateurs artisanaux et affineurs autour d'un enjeu commun: "distinguer le Pélardon d'un produit industriel, se protéger des copies hors zone ", et mettre en avant le terroir. Toutefois, pour préciser le cahier des charges, deux points de vue ont fait débat (Napoléone et al., 2015). Dans le premier, porté essentiellement par des néo-ruraux arrivés à la fin des années soixante-dix l'AOP est un moyen de faire reconnaître des pratiques d'élevage extensif fondées sur le pâturage de parcours souvent embroussaillés. Ces éleveurs se considèrent comme des pasteurs ayant un savoir-faire important dans la maîtrise du pâturage dans ces milieux difficiles et mettent en avant l'intérêt paysager de leurs pratiques. L'autre point de vue était porté par les éleveurs laitiers et les entreprises de transformation. Pour eux, la pratique de référence en élevage est l'alimentation à base d'herbe, à l'auge ou en pâture et souvent issue de prairies temporaires. L'AOP ne devant pas être un facteur d'exclusion de certains éleveurs par un cahier des charges trop restrictif. Ce sont avant tout les atouts commerciaux de l'AOP que ces éleveurs et les transformateurs mettent en avant : protection du produit et accès potentiel à des consommateurs éloignés.

Ce qui a fait accord entre les acteurs est, au final, une fabrication fermière et artisanale. Le cahier des charges retiendra : « lait cru, moulé à la louche, congélation du caillé interdite ». Malgré les difficultés, la coopérative adaptera sa chaîne de transformation pour satisfaire ces clauses. En matière d'élevage, un compromis sera trouvé. La claustration permanente est interdite. Les troupeaux doivent sortir durant 210 jours au pâturage (180 j en altitude). L'enrubannage et l'ensilage sont interdits. Le cahier des charges sera une incitation à l'évolution des pratiques vers le pâturage. Peu à peu, les éleveurs laitiers voient le pâturage comme une ressource alimentaire, au même titre que le foin, et non plus uniquement comme un exercice bénéfique à la santé du troupeau.

La production de Pélardon est essentiellement fermière, sur les 210 tonnes de production, $25 \%$ de la transformation est assurée par la coopérative des Cévennes à Moissac. Dans une situation de très forte concurrence régionale la coopérative a cherché à développer les circuits de la grande distribution nationale. Mais les référencements dans ces enseignes sont difficiles, la logistique est compliquée pour une petite coopérative et les prix sont tirés vers le bas. Depuis 2010, à la faveur de la demande croissante des consommateurs en produits locaux la coopérative a changé d'orientation. Elle met en avant le caractère artisanal de la transformation laitière, la contribution à la vie locale et la dimension familiale de l'élevage. Elle oriente désormais ses circuits commerciaux vers des grossistes indépendants, des détaillants spécialisés et les circuits biologiques.

Dans le contexte local de déprise et de fermeture des paysages les enjeux environnementaux liés à l'élevage concernent prioritairement le maintien de milieux ouverts. Le développement du pâturage dans des secteurs embroussaillés répond aux préoccupations des éleveurs, des élus locaux et des gestionnaires du Parc national des Cévennes. En 2012, « les paysages culturels de l'agro-pastoralisme méditerranéen » des Causses 
et des Cévennes ont été inscrits au patrimoine mondial de l'humanité. Cette reconnaissance du travail séculaire des éleveurs permet d'établir un lien institutionnel entre leur activité et le paysage. Un dispositif de concertation a ensuite été mis en place pour développer une synergie dans la mise en valeur et à la préservation du territoire. Nous sommes bien-là de fait dans un contexte où semble se mettre en place les prémices d'une gestion d'un bien commun complexe associant paysage (Menatti, 2017), élevage et développement local.

\section{Elevage ovin en pays Asses-Verdon-Vaïre-Var}

Le pays Asse-Verdon-Vaïre (pays A3V), centré sur la haute vallée du Verdon est situé entre 800 et $3000 \mathrm{~m}$ d'altitude. La collectivité territoriale du pays et son comité de développement ont, depuis plusieurs années, initié des actions inscrites dans une logique de développement local durable.

L'agriculture ne concerne plus que $8 \%$ des emplois locaux dans un territoire très marqué par les activités touristiques. L'élevage, ovin et secondairement caprin, est la principale activité agricole. Les politiques locales ne sont donc pas orientées sur les seuls intérêts agricoles mais sont pensées dans une logique de développement plus global. Les attentes vis-à-vis de l'agriculture concernent tout d'abord sa capacité à maintenir des actifs, d'où des préoccupations vis-à-vis de la reprise des exploitations. L'aptitude de l'élevage à maîtriser la dynamique de fermeture des paysages par la forêt ainsi que sa contribution à la vie sociale et à la fourniture de produits et services pour l'activité touristique sont également de grande importance.

A l'échelle du pays, la coordination des actions et des acteurs autour de l'agriculture s'est concrétisée dans une charte d'agriculture durable et une charte forestière. Le pays développe en effet différentes actions pour conforter l'élevage et valoriser ses produits auprès des touristes : maison de pays, label " pays gourmand », ouverture du milieu boisé à des fins pastorales. Chacune de ces actions a ses finalités propres et implique des réseaux différents d'acteurs.

L'action pays gourmand a été mise en place en 2011 pour promouvoir les produits agricoles et la culture culinaire locaux. Le pays propose le label «pays gourmand» aux restaurateurs qui s'engagent à promouvoir la culture culinaire locale et à proposer trois plats ou menus composés majoritairement de produits achetés aux producteurs locaux. En 2017 vingt-trois restaurateurs sont engagés dans la démarche. Le Pays Gourmand est un réseau qui rassemble des restaurateurs ainsi que des producteurs et artisans (bouchers) du territoire qui sont leurs fournisseurs. Le pays assure, avec l'appui d'une chargée de mission, l'animation et la promotion de la charte mais procède aussi à la labellisation et à des contrôles du respect de celle-ci.

En matière de gestion de l'espace plusieurs actions sont en cours. Leur ambition est une remise en valeur du foncier pastoral à la fois pour contribuer au maintien du pastoralisme mais aussi dans une perspective de gestion d'un paysage considéré comme contribuant fortement à l'attractivité touristique. Ces actions s'appuient sur la création d'associations foncières pastorales ou d'associations syndicales libres. Elles visent à mobiliser, au-delà des éleveurs, les propriétaires fonciers et à obtenir puis à gérer collectivement des soutiens financiers. Elus locaux et l'Office National des Forêts (ONF) sont également impliqués. 

institutions et un acteur clé assurent un certain lien entre ces actions. Il s'agit tout d'abord de la charte d'agriculture durable et de la charte forestière. Il s'agit ensuite et surtout de la position tutélaire du Pays qui par ses moyens, son dynamisme et sa capacité d'ingénierie réussit à imprimer sa marque dans les différentes actions. Il arrive à influer sur les préoccupations de certains acteurs, comme des élus locaux et l'ONF. Pour autant, la connexion entre les actions de valorisation des produits et celles concernant la gestion productive et paysagère de l'espace n'est pas explicitement formulée, ce qui ne paraît d'ailleurs pas poser de problème. Ainsi les éleveurs engagés dans les actions de valorisation des produits sont rarement les mêmes que ceux qui s'impliquent dans la reconquête pastorale.

\section{Filière Engagement Qualité Carrefour Bleu et Fourme d'Ambert, PNR du Livradois-Forez}

Cette dernière étude de cas correspond à une démarche de certification de la qualité portée par une enseigne de la grande distribution. Elle a été mise en place en 2005 sur le territoire du PNR du Livradois-Forez, en zone de moyenne montagne (Auvergne, France).

L'objectif de cette démarche est de différencier et valoriser des produits issus de systèmes de production et de modes de fabrication spécifiques en contractualisant avec des producteurs et des transformateurs. Cette certification met en avant des conditions de production garantissant une qualité spécifique ainsi que le soutien aux agriculteurs et aux petites et moyennes entreprises agro-alimentaires. Dans le Livradois-Forez, la démarche porte sur deux fromages AOP à pâte persillée : la Fourme d'Ambert et le Bleu d'Auvergne. Elle passe par la mise en place d'une organisation de filière pour garantir l'approvisionnement des magasins du distributeur, en partenariat avec une laiterie basée au cœur du PNR (la seconde du territoire en termes de volume de lait collecté) et une vingtaine d'éleveurs. La laiterie s'engage à collecter le lait des producteurs pendant 7 ans avec un prix majoré. Le distributeur s'engage à acheter un certain volume de fromages à la laiterie et à les vendre dans tous les magasins de France possédant un rayon coupe, à un prix supérieur à celui du produit «standard». Les producteurs, quant à eux doivent respecter le cahier des charges défini par le distributeur et la laiterie. Bien que situé dans le PNR, celui-ci reste un acteur secondaire même s'il suit l'initiative avec attention.

Le cahier des charges proposé aux éleveurs est plus restrictif que celui des AOP sur trois points. Deux d'entre eux concernent la fabrication du fromage. Le troisième a une dimension en lien avec l'environnement: l'alimentation des vaches laitières doit être uniquement composée de fourrages secs et d'herbe éliminant le recours à des fourrages fermentés (ensilage) et au maïs. En 2005, lors du lancement de la filière, le responsable de la laiterie s'est d'abord adressé aux producteurs pratiquant déjà de la sorte ainsi qu'à certains éleveurs en Agriculture Biologique (AB). Même si la culture de l'herbe et du foin reste prégnante sur le territoire, ce ne sont pas des motivations écologiques qui sont derrière l'adhésion des éleveurs mais bien plutôt d'ordre économique, en lien avec la prime proposée.

En termes de valorisation, c'est le distributeur qui est en charge de la commercialisation et de la promotion des produits. La stratégie de certification du distributeur s'inscrit dans une politique de responsabilité sociétale. Cinq valeurs sont affichées par Carrefour : "le goût et l'authenticité des produits ", "un partenariat durable avec les fournisseurs", "un

Géocarrefour, 92/3 | 2018 
prix équitable", "une qualité constante", et "un environnement protégé". Pour la Fourme d'Ambert et le Bleu d'Auvergne, les aspects valorisés par le distributeur via différents medias (site internet notamment) sont l'utilisation de lait cru, la durée d'affinage des fromages et l'alimentation des vaches à base d'herbe et de foin. Ces critères sont associés à une meilleure qualité du lait et à des valeurs d'authenticité des fromages.

Depuis 2005, l'initiative connait des périodes de ralentissement et d'autres de développement (Baritaux et Houdart 2015) selon les débouchés pour les produits sous label «Engagement Qualité Carrefour». Le distributeur, n'est cependant pas le seul acteur à influer sur ce rythme de développement. La laiterie a également un rôle important par ses investissements pour la collecte et la transformation du lait et par le recrutement d'éleveurs. Enfin, les relations entre ces différents acteurs restent complexes avec des périodes de confiance où l'existence d'intérêts convergents est affirmée par tous alors qu'à d'autres moments ce sont l'incompatibilité des objectifs de chaque partie prenante et les divergences de conception sur les pratiques à mettre en œuvre qui prédominent.

\section{Discussion}

\section{Un écheveau d'actions collectives qui restent peu connectées}

Dans les quatre études de cas analysées, il n'y a pas d'action collective envisageant l'élevage dans sa globalité mais il existe de fait des relations entre les instances se préoccupant des espaces pastoraux et celles dédiées à la valorisation des produits. La situation est celle d'un écheveau d'actions collectives plus ou moins connectées. Dans tous les cas, les systèmes d'acteurs sont complexes avec plusieurs lieux de gouvernance regroupant des acteurs différents autour de finalités différentes. Trois grands types de lieux ont été identifiés. Tout d'abord ceux réunissant les éleveurs et les acteurs économiques de l'aval engagés dans la transformation et la valorisation des produits. Ensuite, ceux mobilisant les acteurs de l'environnement, des collectivités territoriales et de l'Etat autour de la gestion de l'environnement dans l'espace pastoral. Souvent quelques éleveurs y sont également présents. Enfin, techniciens et conseillers agricoles accompagnés de responsables professionnels de l'élevage échangent de leur côté pour produire des conseils techniques sur l'alimentation des animaux et la conduite productive des surfaces pastorales.

Les liens entre ces différents lieux restent peu institutionnalisés. Ce qui les relie, ce sont tout d'abord des acteurs, responsables professionnels agricoles, agents des collectivités territoriales en particulier du Parc naturel régional du Vercors et du Livradois-Forez qui s'impliquent dans plusieurs actions. Ils portent d'un lieu à un autre des éléments de cohérence, parviennent à exprimer une complémentarité entre les actions et les préoccupations. Dans d'autres cas, ce sont des agréments partagés et reconnus comme la charte d'agriculture durable dans le pays Asses-Verdon-Vaïre-Var, ou dans les Cévennes l'inscription des paysages culturels de l'agro-pastoralisme au patrimoine mondial de l'humanité qui incitent à la rencontre et à la recherche d'une cohérence. Enfin, certains objets, comme le Bleu du Vercors, le Pélardon ou encore les paysages et les espaces pastoraux créent aussi du liant car ils sont discutés voire réappropriés de façon variée dans différentes instances. 
41 Au-delà de la séparation standard entre acteurs économiques et acteurs environnementaux les spécificités des problèmes à gérer expliquent aussi cette pluralité des lieux de gouvernance. En matière d'environnement, de préservation de la biodiversité par exemple, toutes les agricultures et toutes les productions sont potentiellement concernées et pas seulement celles engagées dans une démarche de valorisation des produits. D'un côté il s'agit de fédérer des producteurs autour d'un projet de valorisation de leur production, de l'autre il importe d'obtenir une synergie entre agriculteurs utilisant le même espace. Pour autant, cette division contribue à maintenir une ségrégation entre préoccupations environnementales et productives.

Ainsi dans les initiatives où la priorité est la valorisation et la différenciation des produits, la prise en compte de préoccupations environnementales reste souvent indirecte sans référence aux enjeux et aux actions environnementales concernant l'élevage du territoire. Les acteurs en présence sont essentiellement économiques: éleveurs, coopératives, entreprises de transformation et de distribution, de la multinationale au petit restaurateur local. Ils sont souvent appuyés par les collectivités territoriales mais sont rarement en relation avec des acteurs de l'environnement. Leurs finalités relèvent très majoritairement d'un maintien ou d'un développement des capacités productives de l'agriculture: pérenniser le tissu d'exploitations agricoles, améliorer le revenu des agriculteurs, etc. Ces objectifs sont souvent resitués dans une perspective plus large de développement territorial et en matière environnementale, ce sont seulement des avantages liés à la territorialisation qui sont évoqués : valorisation et préservation des ressources locales, maintien de la qualité des paysages, réduction des trajets et conséquences positives sur la consommation d'énergies fossiles, etc. Les enjeux de préservation de la biodiversité ou de la qualité de l'eau portés par les acteurs environnementaux sont peu présents dans les débats. De façon inverse, les difficultés à réussir une démarche de valorisation des produits restent peu évoquées par le monde de l'environnement qui a souvent un discours se limitant à une vision idéalisée des circuits courts.

\section{Actions collectives, gestion des ressources naturelles et valorisation des produits de l'élevage}

43 La mobilisation de la théorie des communs pour analyser les transformations de l'élevage dans les territoires permet d'aller plus loin que les constats du paragraphe précédent en regardant la nature et les conditions de construction d'arrangements entre acteurs.

Ce sont tout d'abord la prise en compte de fonctions et d'attentes nouvelles pour l'élevage qui motive la mise en place des différentes formes de gouvernance collective observées. Ces fonctions s'inscrivent dans une perspective d'amélioration des contributions de l'élevage au développement local. Elles concernent la fourniture de produits alimentaire de qualité, la préservation des paysages et de l'environnement, la vie sociale et économique des territoires. Ces fonctions n'ont d'ailleurs pas de spécificité particulière dans les territoires étudiés et sont assez fréquemment identifiés à l'échelle européenne (Dumont et Dupraz, 2016). Pour autant, elles deviennent de plus en plus prégnantes et aujourd'hui les frontières entre rationalité territoriale de différenciation des produits et rationalité critique fondée sur la mise en avant d'avantages environnementaux, sociaux et économiques des conditions de productions de certains produits (Ilbéry et al., 2005) paraissent de moins en moins marquées. 

collectives qui se mettent en place sont liées à l'apparition de nouveaux acteurs qui sont de plus en plus présents et influents : l'Etat et l'Europe, les collectivités territoriales qui sont très puissantes dans nos études de cas (Parcs régionaux, intercommunalités), les acteurs de l'environnement et des filières. Les éleveurs restent certes les bénéficiaires immédiats de l'élevage mais même si ces autres acteurs en sont des bénéficiaires indirects ils pèsent de plus en plus dans les débats sur ce que doivent être l'élevage et ses pratiques. Injonctions d'ailleurs parfois contradictoires, comme il en est dans le Vercors, où les environnementalistes mettent en avant la biodiversité des prairies permanentes pour justifier des pratiques extensives alors que les conseillers d'élevage chargés de veiller à la qualité du lait leur recommandent de recourir aux prairies temporaires pour la qualité laitière de leur fourrage. Même si les controverses demeurent progressivement des accords se passent et ce qui se joue c'est bien la construction de normes qui définissent le métier d'éleveur et orientent ceux-ci dans la configuration de leurs systèmes d'élevage et la conduite de leurs troupeaux.

Certes ces accords ont bien le statut de constructions négociées mais les arrangements qui en résultent traduisent des hiérarchies entre acteurs. Hiérarchies entre acteurs non agricoles et éleveurs comme nous venons de le voir mais aussi entre éleveurs. Il en a été ainsi lors de l'élaboration des cahiers des charges des AOP Bleu du Vercors Sassenage et Pélardon. Il en est encore ainsi lorsqu'il s'agit de réviser ces cahiers des charges. Ce sont des périodes durant lesquelles les tensions entre éleveurs sont souvent très fortes. Les différences de conception dans ce que doivent être l'élevage et ses produits s'expriment fortement. Même si dans ces deux territoires la volonté de ne pas exclure reste prégnante, les compromis sont difficiles. Certains éleveurs doivent accepter de fortes adaptations. Il en est ainsi des producteurs fermiers du Vercors ou de la coopérative de Moissac dans les Cévennes qui a dû modifier sa chaine de transformation pour transformer du lait cru moulé à la louche). L'appropriation des ressources est au cœur des enjeux (Linck, 2007), il y a de fait une politisation de l'élevage, il fait l'objet de revendications et les histoires de la construction des arrangements que nous avons retracées invitent à dépasser une vision idéalisée du collectif et de la participation.

\section{Conclusion}

Les quatre études de cas de cet article montrent une réalité contrastée. Au-delà et en contrepoint d'une gestion en biens privés, dans l'exploitation agricole comme dans les entreprises privées et les coopératives, on y observe des modes de gestion collectifs polycentriques de l'élevage avec d'un côté actions de valorisation des produits et de l'autre, actions environnementales. Ce sont ces formes d'action collective qui font l'objet des normes et des règles les plus formalisées par contraste avec des formes nouvelles contribuant à l'intégration des fonctions productives, environnementales et alimentaires qui ont également été observées. Ces dernières reposent soit sur des acteurs présents dans différents lieux, comme certains agents des parcs naturels régionaux et nationaux soit sur des accords peu institutionnalisés, par exemple des chartes. Ce qui est en jeu dans ces différents arrangements est bien l'avenir de ce que doivent être l'élevage et ses pratiques.

Mais pour autant peut-on parler de gestion territoriale de l'élevage en bien commun complexe? Pour discuter cette question nous avons cherché à dépasser le résultat des

Géocarrefour, 92/3 | 2018 
processus de l'action collective, les règles et les normes, sur lequel se focalise la théorie des communs pour nous intéresser à la dynamique de ces processus. Nous avons adopté une approche constructiviste des biens communs et de l'action collective. Les constructions des arrangements mixtes rendent compte ainsi non seulement de la diversité des fonctions attachées à l'élevage mais aussi de controverses et de hiérarchies entre acteurs, entre éleveurs tout d'abord mais aussi entre éleveurs et acteurs internes (collectivités, associations....), comme extérieurs au territoire et souvent puissants (Etat, grande distribution...). Pour autant des collectifs se forment et s'organisent pour prendre en charge différentes fonctions de l'élevage. Ils doivent chercher à concilier interventions publiques, mécanismes marchands et rapports de pouvoir entre acteurs et prennent un caractère hybride. Cette gestion émergente en bien commun complexe est bien le fruit de contraintes, d'intérêts, de valeurs et de niveaux de pouvoir différents. Les accords qui en résultent sont provisoires et à réviser régulièrement. Ces accords, sous prétexte qu'ils seraient le fruit d'une construction collective ne doivent pas être idéalisés et nous restons souvent assez loin d'une action collective harmonieuse autour de la gestion de ressources partagées et d'intérêts collectifs bien compris.

\section{BIBLIOGRAPHIE}

BARITAUX V. et HOUDART M., 2015, Relations fournisseurs-grande distribution dans les filières agroalimentaires. Une analyse de la trajectoire d'une démarche «filière qualité », Économie rurale, n³46, p. 15-30.

BARITAUX V., HOUDART M., BOUTONNET J.-P., CHAZOULE C., CORNIAUX C., FLEURY P., LACOMBE N., NAPOLÉONE M., TOURRAND J.-F., 2016, Ecological embeddedness in animal food systems (re-)localisation: A comparative analysis of initiatives in France, Morocco and Senegal, Journal of Rural Studies, Vol. 43, p. 13-26.

BARON C., PETIT O., ROMAGNY B., 2011, Le courant des « Common-Pool Resources » : un bilan critique, in T. DAHOU, M. ELLOUMI F. MOLLE, M. GASSAB et B. ROMAGNY (dir.), Pouvoirs, Sociétés et Nature au Sud de la Méditerranée, Paris/Tunis, Editions INRAT/IRD/Karthala, p. 29-51.

BERGE E., 2011, Editorial; Governing the Commons for two decades: a complex story, International Journal of the Commons, Vol. 5., n², p. 160-187.

CLEMENT F., 2013, For critical social-ecological system studies: Integrating power and discourses to move beyond the right institutional fit, Environmental Conservation, Vol 40., n², p. 1-4.

DIETZ T., OSTROM E., STERN P.C., 2003, The Struggle to Govern the Commons, Science, Vol. 302, ${ }^{\circ}$ 5652, p. 1907-1912.

DARDOT P. et LAVAL C., 2014, Commun. Essai sur la révolution au XXI siècle, Paris, La Découverte, $600 \mathrm{p}$.

De MOOR T., 2011, From common pastures to global commons: a historical perspective on interdisciplinary approaches to commons ", Natures Sciences Sociétés, Vol. 19, n 4, p. 422-431. 
DUMONT B. et DUPRAZ P. (coord.), 2016, Rôles, impacts et services issus des élevages en Europe. Synthèse de l'expertise scientifique collective, Paris, INRA éd., 133 p.

FAO, 2009, La situation mondiale de l'alimentation et de l'agriculture. Le point sur l'élevage, Rome, FAO, $202 \mathrm{p}$.

FOURNIER S., BIÉNABE E., MARIE-VIVIEN D., DURAND C., SAUTIER D., CERDAN C., 2018, Les indications géographiques au regard de la théorie des communs, Revue internationale des études du développement, Vol. 233, n¹, p. 139-162.

HARDIN G., 1968, The Tragedy of the Commons. Science, Vol. 162, n³859, p. 1243-1248.

HESS C., and OSTROM E., 2006, Understanding Knowledge as a Commons. From Theory to Practice, Cambridge, MA, MIT Press, $382 \mathrm{p}$.

ILBERY B., MORRIS C., BULLER H., MAYE D., KNEAFSEY M., 2005, Product, process and place: an examination of food marketing and labelling schemes in Europe and North America, European Urban and Regional Studies, Vol. 12, n², p. 116-132.

LAMINE C., et CHIFFOLEAU Y., 2012, Reconnecter agriculture et alimentation dans les territoires : dynamiques et défis, Pour, Vol. 215-216, p. 85-92.

LINCK T., 2007, Patrimoines sous tension. L'exclusion, condition et écueil de l'appropriation collective, Économie appliquée, Vol. 3, p. 177-198.

MARSHALL G. R., 2015, A social-ecological systems framework for food systems research: accommodating transformation systems and their products, International Journal of the Commons, Vol. 9 (2), p. 881-908.

MENATTI L., 2017, Landscape: from common good to human right, International Journal of the Commons, Vol. 11, n 2, p. 641-683.

MOSSE D., 2008, Collective action, common property, and social capital in South India: An anthropological commentary, in BARDHAN P. et RAY I., (ed.), The Contested Commons, New Delhi, Oxford University Press, p. 695-724.

NAPOLÉONE M., CHAZOULE C. FLEURY P. 2015, La ressource fourragère qui convient, Techniques et Culture, Vol. 63, p. 110-129.

OSTROM E., 1990, Governing the commons. The evolution of institutions for collective action, Cambridge, Cambridge University Press, $271 \mathrm{p}$.

OSTROM E., 2008, Institutions and the environment, Economics affairs, Vol. 28, n³, p. 24-31

OTTE J., COSTALES A., DIJKMAN J., PICA-CIAMARRA U., ROBINSON T., AHUJA V., LY, C., ROLANDHOLST D., 2012, Livestock sector development for poverty reduction: an economic and policy perspective Livestock's many virtues, Rome, FAO, $161 \mathrm{p}$.

POTEETE A R., JANSSEN M. A., OSTROM E. (eds.), 2010, Working together: collective action, the commons, and multiple methods and practice, Princeton, Princeton University Press, $376 \mathrm{p}$.

RUBINO R., SEPE L., DIMITRIADOU A., GIBON A., 2006, Livestock farming systems: Product quality based on local resources leading to improved sustainability, Wageningen, Wageningen Academic Publishers, $410 \mathrm{p}$.

STEINFELD H., GERBER P., WASSENAAR T., CASTEL V., ROSALES M., DE HAAN C., 2006, Livestock's Long Shadow. Environmental issues and options, Rome, FAO Report, 380 p. 
STOJANOVIC T., MCNAE H., TETT P., POTTS T.W., REIS J., SMITH H.D., DILLINGHAM I., 2016, The "social" aspect of social-ecological systems: a critique of analytical frameworks and findings from a multisite study of coastal sustainability, Ecology and Society, Vol. 21, n³, art 15.

VAN DER PLOEG J. D., JINGZHONG Y., SCHNEIDER S., 2012, Rural development through the construction of new, nested, markets: comparative perspectives from China, Brazil and the European Union, Journal of Peasant Studies, Vol. 39, n¹, p. 133-173.

WILSON D. C. K., 2012, European mixed fisheries as a complex commons, Natures Sciences Sociétés, Vol 20, p. 66-74.

\section{RÉSUMÉS}

Cet article propose une analyse des mutations des liens entre élevage et territoire. A l'aide de la théorie des communs nous cherchons à voir s'il existe des formes de gouvernance de l'élevage en bien commun complexe traitant globalement de la valorisation des produits et de l'environnement. Quatre études de cas, dans des territoires de montagne français, montrent une réalité contrastée. On y observe des modes de gestion polycentriques de l'élevage, avec d'un côté actions de valorisation des produits et de l'autre, actions environnementales. Des formes nouvelles contribuant à l'intégration de ces fonctions ont été observées. Elles reposent sur des acteurs présents dans différents lieux ou sur des accords peu institutionnalisés (chartes). Il y a là des formes émergentes de gouvernance de l'élevage en bien commun complexe. Il existe cependant des controverses et des hiérarchies entre éleveurs et entre mondes agricole et non agricole qui invitent à ne pas idéaliser ces formes d'action collective.

This paper proposes an analysis of the mutations in the links between the livestock sector and its related territory. With reference to the "commons theory", the article aims to show whether in some cases the livestock sector is managed as a complex "commons", aggregating the enhancement of products and preservation of the environment. Four case studies in the French mountainous territories show the contrast between different realities. These include the polycentric management of livestock with on the one hand actions towards enhancing the value of products and, on the other, actions focused on protecting the environment. New forms of governance contributing to the integration of these functions have been observed. They are based on actors present in different places or on weakly institutionalized agreements (charters). These are emerging forms of governance of livestock farming as a complex "commons" good. However, controversies and social hierarchies exist between livestock farmers and between farmers and non-farmers suggesting that these forms of collective action should not be idealised.

\section{INDEX}

Keywords : shared resources, collective action, territory, livestock farming, environment, highquality food systems

Mots-clés : ressources communes, action collective, territoire, élevage, environnement, systèmes alimentaires de qualité 


\section{AUTEURS}

\section{PHILIPPE FLEURY}

ISARA-Lyon, AGRAPOLE - 23 rue Jean Baldassini, F - 69364 Lyon Cedex 07, pfleury@isara.fr

\section{MARIE HOUDART}

IRSTEA, UMR Metafort, 24, avenue des Landais, CS 20085, 63178 Aubière, marie.houdart@irstea.fr

\section{JACQUES LASSEUR}

INRA, SELMET, 2 place Viala 34060 Montpellier Cedex 2, jacques.lasseur@inra.fr

\section{VIRGINIE BARITAUX}

Clermont Université, VetAgro Sup, UMR Métafort, F-63370 Lempdes, virginie.baritaux@vetagrosup.fr

\section{CAROLE CHAZOULE}

ISARA-Lyon, AGRAPOLE - 23 rue Jean Baldassini, F - 69364 Lyon Cedex 07, chazoule@isara.fr Christian Corniaux CIRAD, ISRA/LNERV Pôle PPZS, BP 2057, Dakar, Sénégal, christian.corniaux@cirad.fr

\section{MARTINE NAPOLÉONE}

INRA, SELMET, 2 place Viala 34060 MONTPELLIER CEDEX 2, martine.napoleone@inra.fr

\section{RENÉ POCCARD-CHAPUIS}

CIRAD, Brésil rene.poccard-chapuis@cirad.fr 